\title{
Interventions to Improve Decision Making and Reduce Racial and Ethnic Disparities in the Management of Prostate Cancer: A Systematic Review
}

\author{
Saleha Sajid, $M D^{1,2}$, Ashwin A. Kotwal, $B A^{7}$, and William Dale, $M D, P h D^{1,2}$ \\ 'Department of Medicine, Section of Geriatrics, \& Palliative Medicine, University of Chicago Medical Center, Chicago, IL, USA; ${ }^{2}$ Department of \\ Medicine, Section of Hematology/Oncology, University of Chicago, Chicago, USA.
}

\begin{abstract}
OBJECTIVES: Ethnic minorities are disproportionately impacted by prostate cancer $(\mathrm{PCa})$ and are at risk for not receiving informed decision making (IDM). We conducted a systematic literature review on interventions to improve: (1) IDM about PCa in screeningeligible minority men, and (2) quality of life (QOL) in minority PCa survivors.
\end{abstract}

DATA SOURCES: MeSH headings for $\mathrm{PCa}$, ethnic minorities, and interventions were searched in MEDLINE, Cochrane Database of Systematic Reviews, Cochrane Central Register of Controlled Trials, CINAHL, and PsycINFO.

SUBJECT ELIGIBILITY CRITERIA: We identified U.S.based, English-language articles (1985-2010) on interventions to improve PCa IDM and QOL that included $50 \%$ or more minority patients or analyses stratified by race/ethnicity.

STUDY APPRAISAL AND SYNTHESIS METHODS: Articles $(n=19)$ were evaluated and scored for quality using a Downs and Black (DB) system. Interventions were organized by those enhancing 1) IDM about PCa screening and 2) improving $\mathrm{QOL}$ and symptom among PCa survivors. Outcomes were reported by intervention type (educational seminar, printed material, telephonebased, video and web-based).

RESULTS: Fourteen studies evaluated interventions for enhancing IDM about PCa screening and five evaluated programs to improve outcomes for PCa survivors. Knowledge scores were statistically significantly increased in 12 of 13 screening studies that measured knowledge, with ranges of effect varying across intervention types: educational programs (13\%-48 \% increase), print (11\%-18\%), videotape/DVD (16\%), and web-based (7 \%-20\%). In the final screening study, an intervention to improve decision-making about screening increased decisional self-efficacy by $9 \%$. Five cognitive-behavioral interventions improved $\mathrm{QOL}$ among minority men being treated for localized PCa through enhancing problem solving and coping skills.

LIMITATIONS: Weak study designs, small sample sizes, selection biases, and variation in follow-up intervals across studies.

CONCLUSIONS: Educational programs were the most effective intervention for improving knowledge among

Systematic review registration number $N / A$ screening-eligible minority men. Cognitive behavioral strategies improved QOL for minority men treated for localized PCa.

KEY WORDS: prostate cancer; interventions; informed decision making; disparities; African American.

$\mathrm{J}$ Gen Intern Med 27(8):1068-78

DOI: $10.1007 / \mathrm{s} 11606-012-2086-5$

(C) Society of General Internal Medicine 2012

\section{INTRODUCTION}

Prostate cancer (PCa) is a major cause of morbidity and mortality for men in the United States. In 2010, an estimated 217,730 new cases were diagnosed and over 30,000 men died of prostate cancer. ${ }^{1}$ African American (AA) men have lower rates of early detection and increased mortality compared to non-Hispanic whites. ${ }^{2}$ These stark statistics led to increased PCa screening for all men with the introduction of serum prostate specific antigen (PSA) testing in the late 1980s. However, screening with PSA has been controversial since its introduction, due to uncertainty about the impact of PSA screening in reducing mortality, ${ }^{3}$ its non-specificity for $\mathrm{PCa}$, and its inability to gauge PCa aggressiveness. ${ }^{4}$ Recommendations have shifted from encouraging PSA screening ${ }^{5}$ to discouraging it as the standard of care. ${ }^{6}$

Over the past 25 years, shared decision making between patient and physician has been increasingly emphasized, especially for PCa. In 1992, the American Cancer Society recommended that all men $\geq 50$ undergo PSA testing annually. ${ }^{5}$ In contrast, in 1989, the United States Preventive Services Task Force (USPSTF) recommended routine PCa screening for men with a life expectancy $>10$ years and encouraged patients who requested screening to be given objective information about the benefits and harms of early detection and treatment. ${ }^{7}$ Recently updated USPSTF guidelines now recommend against routine PSA screening in all age groups, giving it a grade $\mathrm{D}$ recommendation, and concluding with "moderate certainty" that the harms of $\mathrm{PCa}$ screening outweigh the benefits. ${ }^{6}$ However, USPSTF guide- 
lines do not directly address racial and ethnic disparities in $\mathrm{PCa}$, possibly underestimating the lost benefits of discontinuing testing in high-risk ethnic groups, specifically in AA men. ${ }^{8,9}$ Despite these shifting guidelines, the call for informed decision making (IDM) for all men, including ethnic minorities, has remained a constant. ${ }^{10}$

IDM is important for minorities, such as AA men, who are disproportionately affected by PCa. AA men are nearly $60 \%$ more likely to be diagnosed with $\mathrm{PCa}$ and more than twice as likely to die from it compared to white men. ${ }^{11}$ Physicians are less likely to discuss treatments with AA men compared to white men, ${ }^{12}$ and cultural barriers may prevent AA men from seeking emotional support regarding their PCa. ${ }^{13}$ This suboptimal communication can lead to increased decisional conflict, increased anxiety, and poorer quality of life (QOL). Tillman has argued that reducing racial-ethnic disparities should focus on instituting a wellcoordinated system of care that provides high quality interventions focused on culturally-sensitive shared decision making, defined by recognizing ethnicity and culture as a central structure of the intervention. ${ }^{14}$ In addition, psychological interventions focusing on cancer-related stress and QOL could help narrow the racial disparities in $\mathrm{PCa}$ management. ${ }^{15}$

To better understand what is known about the management of PCa for minorities, we conducted a systematic review of the literature summarizing interventions to improve informed decision making about $\mathrm{PCa}$ detection among screening-eligible minorities, and QOL among minority $\mathrm{PCa}$ survivors.

\section{METHODS}

We followed the guidelines of the Preferred Reporting Items for Systematic Reviews and Meta-Analyses (PRISMA) statement. $^{16}$

\section{Initial Search}

We searched MEDLINE via Ovid using specific Medical Subject Heading (MeSH) terms. We combined MeSH terms referring to $\mathrm{PCa}$, racial/ethnic minorities, and healthcare disparities. The specific terms included in the search strategy were: [(Prostatic neoplasms/prostate cancer or prostate specific antigen or psa or digital rectal examination or dre) AND [(Race or racial or minority or African Americans or Hispanic Americans or Asian Americans or Indians, North American or Continental Population Groups or African continental ancestry group or American native continental ancestry group or Asian continental ancestry group or oceanic ancestry group or brazil [United States] or Portugal [United States] or Arabs[United State, or Inuits)] OR [(Healthcare disparities or health status disparities, or minority health or health*adj3disparit* or underserved)]
AND [(Intervention or intervention studies or health interventions or health knowledge)] limited to English language, humans and adults. We conducted parallel searches in the Cochrane Database of Systematic Reviews, Cochrane Central Register of Controlled Trials, the Cumulative Index of Nursing and Allied Health Literature (CINAHL) and PsycInfo. We then combined search results from all databases and removed duplicate articles.

\section{Inclusion/Exclusion Criteria}

We included studies on humans, in English language that were conducted in the US and published between 1985 and 2010. Articles had to include at least one ethnic minority group (overall patient population more than $50 \%$ minority or, if less than $50 \%$ minority, include specific analysis by race/ethnicity) and an IDM or QOL-affecting intervention taking place within an identified health care setting. Community-based interventions linked to a consistent source of health care delivery were included. The initial search yielded 2,815 articles (Fig. 1). In light of current recommendations, studies that assessed only screening behavior, without consideration of IDM or QOL, were excluded.

\section{Article Selection (Figure 1)}

Two primary reviewers (SS, AK) independently evaluated each article for inclusion. Disagreements in articles selected were discussed between the reviewers, with input from a third reviewer (WD) as necessary for adjudication. Only articles that explicitly included interventions were selected for further review $(n=76)$. From these articles, abstracts were blindly and independently reviewed by SS and AK; full articles were reviewed in the event of disagreements. "Pre-intervention" studies, in which interventions were being developed or tested only for feasibility, were excluded. Articles exclusively studying screening behavior (e.g. changes in PSA screening rates) without inclusion of some aspect of IDM or QOL as an outcome were removed $(n=3)$. Following selection $(n=12)$, each full article was independently evaluated by the primary reviewers. Review of references of these articles added 7 more studies to the total $(n=19)$.

\section{Quality and Bias Assessment}

All papers were evaluated for methodological quality using the Downs and Black (DB) scoring algorithm (0 to 28), which allows assessment of articles across various study designs. ${ }^{17}$ Methods are described in detail elsewhere. ${ }^{18}$ In brief, DB scoring rates each study based on external validity, bias, confounding, and power. We also added one 


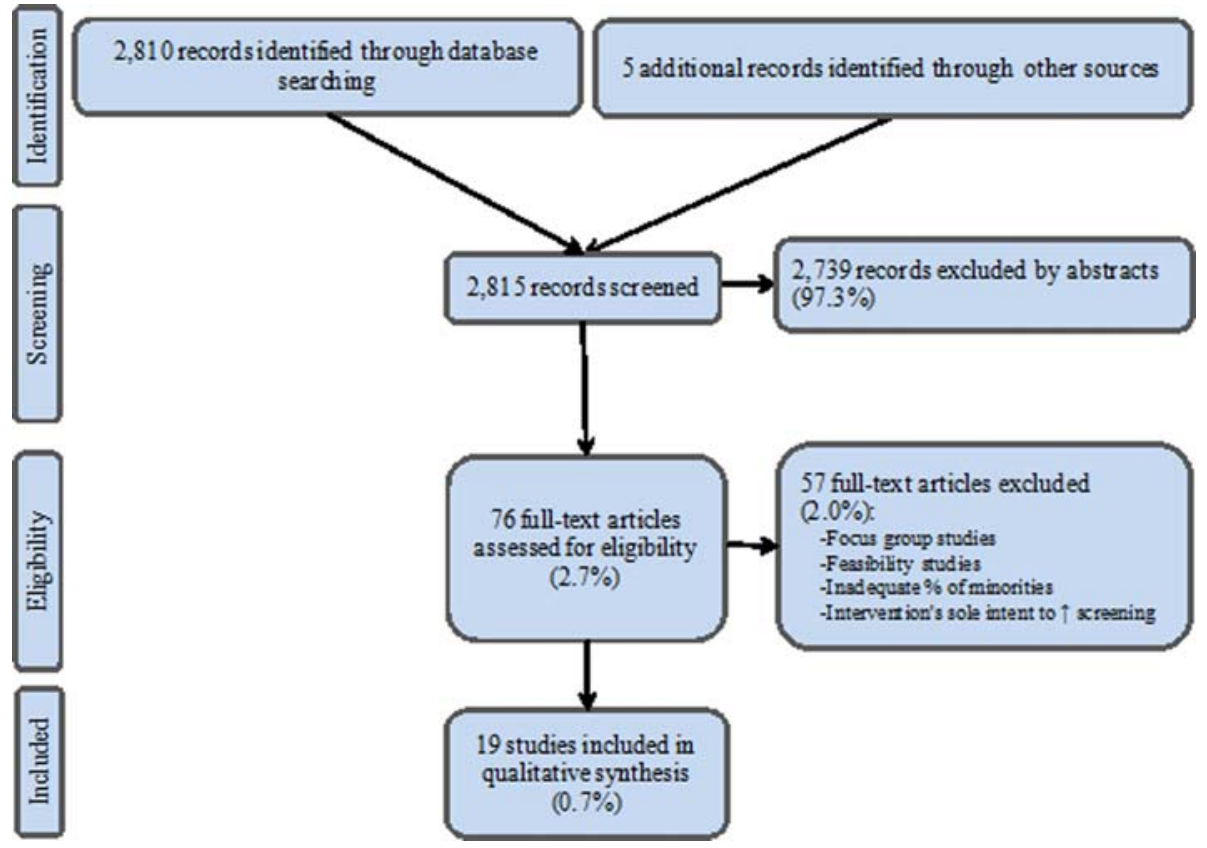

Figure 1. Flow diagram of the inclusion/exclusion of studies for review.

item from the Cochrane tool for bias $^{19}$ that was not captured with the DB tool, for a total maximum modified DB score of 29. DB score ranges were grouped for randomized studies into the following four quality levels: very good ( $\geq 20)$, good (15 to 19$)$, fair (11 to 14 ) and poor (10 or less) ${ }^{20}$ We did not assess for a risk of publication bias.

\section{Data Extraction and Synthesis (Tables 1, 2)}

The results are organized slightly differently between the tables and text. To prevent multiple listing of studies, the tables are organized as follows. First, the 19 studies are divided into screening studies (Table 1) and PCa survivor studies (Table 2). Second, within each type of study, papers are grouped by intervention type: 1) educational program, 2) printed-material/booklets, 3) telephone/videotape/DVD, and 4) web-based. Some studies have multiple types of interventions, but they are listed in the area that is the dominant intervention. Third, the specific outcome measures (e.g.- -knowledge and self-efficacy for IDM in screening-eligible men; QOL and symptom management self-efficacy among PCa survivors) are described in the "outcomes" column. To complete the tables, studies are compared based on study design, sample size, ethnicity of population, study duration, follow-up period, content of intervention, results, and Cochrane-modified DB quality score.

To report the findings in a way that is most clinically meaningful, the text is organized as follows.
First, as in the table, we discuss screening studies and survivorship studies separately. Second, we discuss outcomes associated with each type of study. For screening studies, we describe knowledge about $\mathrm{PCa}$ and screening self-efficacy. For survivorship studies, we report QOL and self-efficacy about symptom management. Third, we discuss the results, within each outcome, based on the intervention used (e.g. educational program or printed materials).

Finally, in order to compare results across different intervention types for the same outcome such as "knowledge", we calculated a "percent change" value for that outcome. That is, for studies that found statistically significant differences in that outcome across intervention groups, we calculated and reported a "percent change/ difference" in the relevant score. While we cannot report pvalues of significance for these percentages, they provide a range of values that can be considered for assessing the impact of interventions across studies for specific outcomes. The final set of articles varied significantly in outcome reporting, use of controls, and risk adjustment, so a metaanalysis was not possible.

\section{RESULTS}

Overall, 14 of the 19 studies were of screening-eligible men being evaluated for IDM (Table 1), and the remaining 5 were on improving outcomes for PCa survivors (Table 2). The Cochrane-modified DB scores for studies varied across intervention types as follows: (1) educational seminar interventions, $19.3 \pm \mathrm{SD} 3.5$; (2) printed material/booklet 







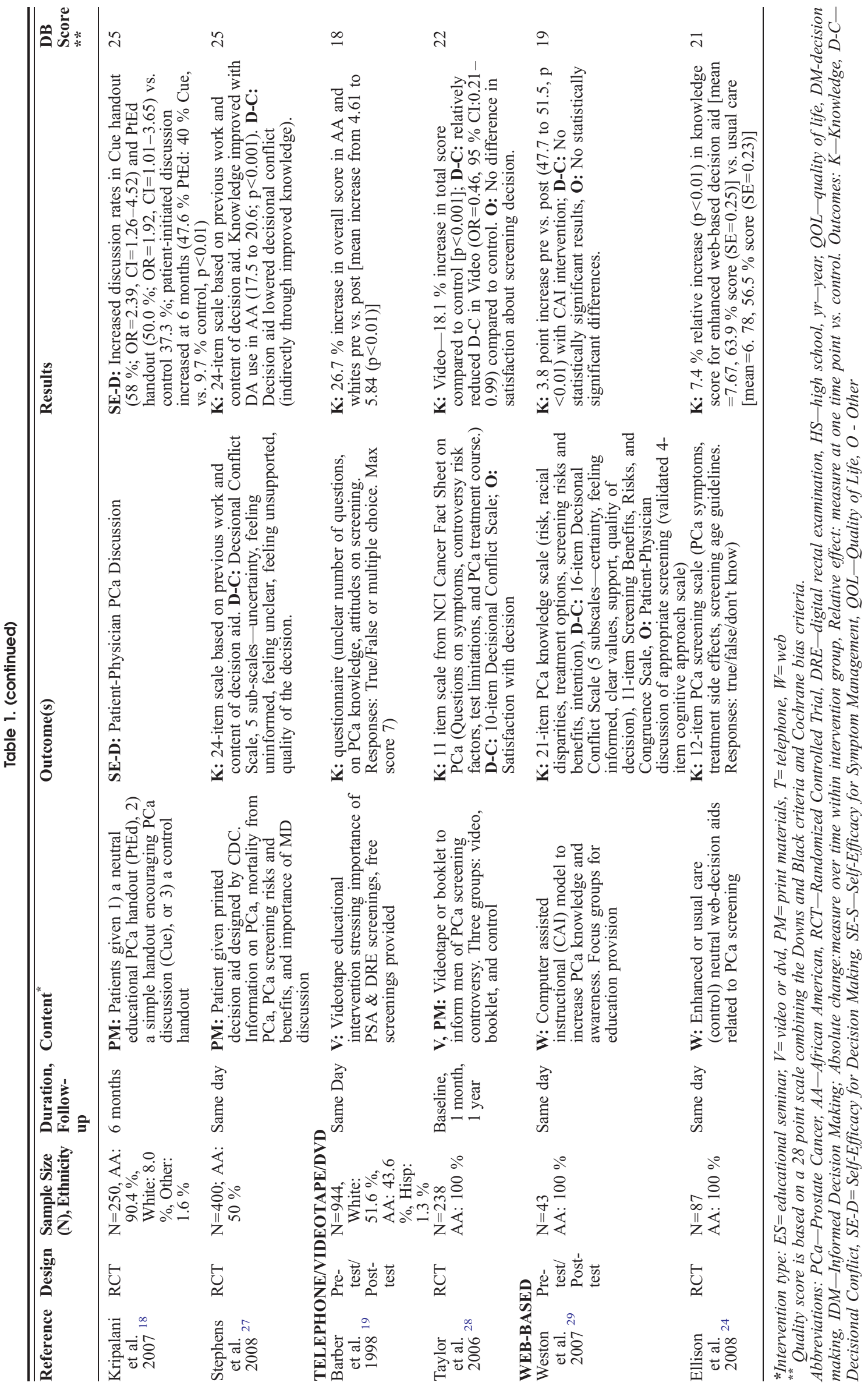




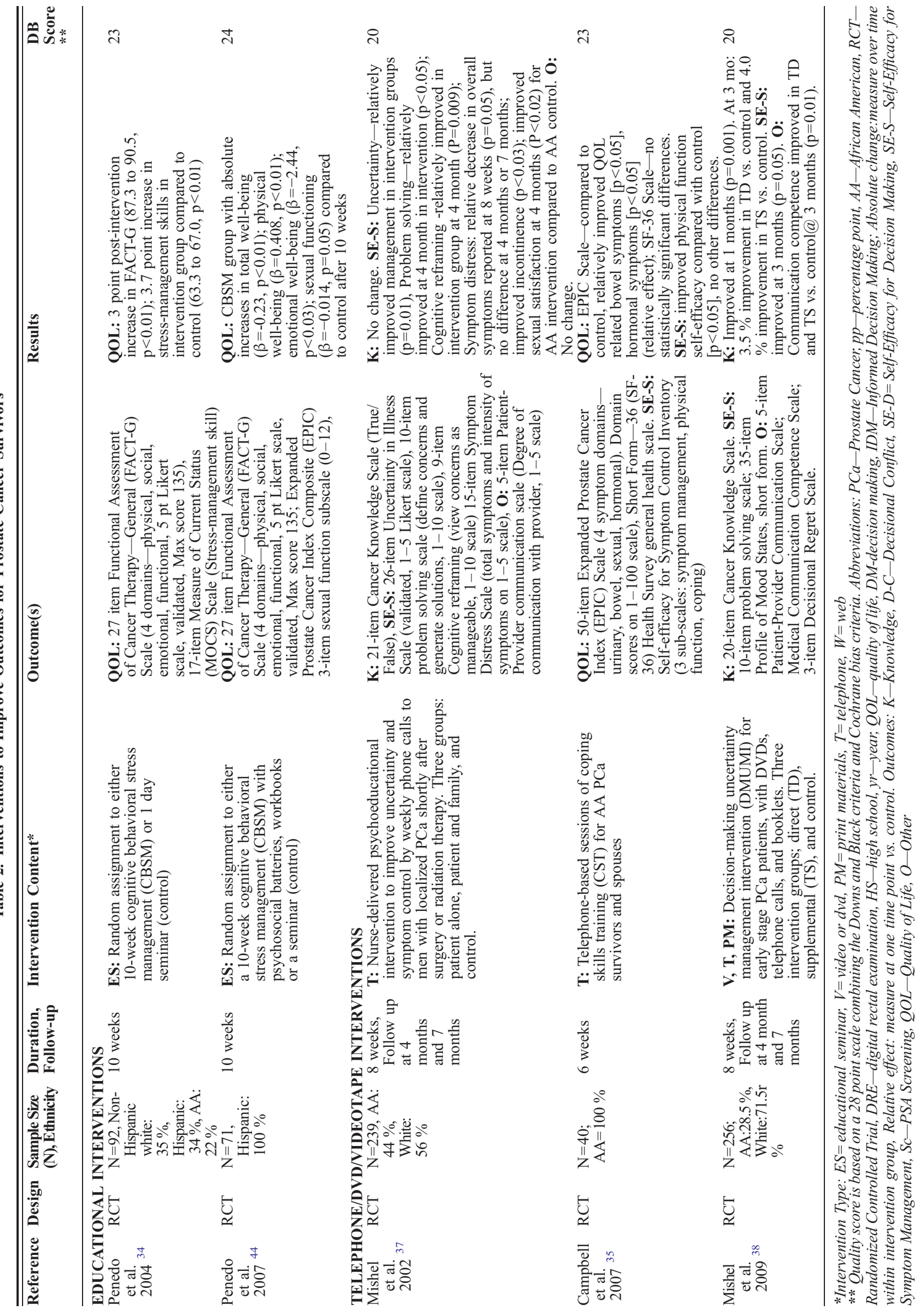


interventions, $22.6 \pm \mathrm{SD} 1.9$; (3) telephone-based interventions, 21.0 \pm SD 1.7; (4) video-tape/DVD interventions, 20.3 \pm SD 1.7; and (5) web-based interventions, $20.0 \pm$ SD 1.4, consistent with "good" to "very good" scores.

\section{Interventions to Enhance Informed Decision Making for Prostate Cancer Screening (Table 1)}

Prostate Cancer Knowledge (n=13 Across Intervention Types). Of the 14 studies of PCa screening, 13 assessed knowledge changes, most using same day assessments. An additional screening study did not evaluate changes in knowledge at all and is described in the next section. ${ }^{18}$. $\mathrm{PCa}$ knowledge scores increased in 12 studies ${ }^{19-30}$ and did not change in $1 .{ }^{31}$ Five were RCTs, ${ }^{24,25,27,28,31} 7$ were pre-/ post-test studies, ${ }^{19-23,29,30}$ and 1 a cohort study. ${ }^{26}$ Eight assessed the effects of educational programs, ${ }^{20-23,25,26,30,31}$ 2 printed materials/booklets, ${ }^{27,28} 1$ a videotape/DVD, ${ }^{19}$ and 2 web-based interventions. ${ }^{24,29}$ Overall, AA men represented $84 \%$ of the study population across the studies.

Educational Programs $(\boldsymbol{n}=8)$. PCa knowledge increased in 7 of these studies. Educational programs included combinations of lectures provided by trained experts, primarily people in the community trained by specialists, and/or didactic seminars. Regardless of the source for the questions asked, men were typically provided a basic understanding of $\mathrm{PCa}$ risk factors, and they were explained the risk, benefits and limitations of available screening and treatment modalities.

Two RCTs tested educational programs. Holt et al built on prior work with AA women (in which a spirituality-based approach was used to encourage mammography), randomly assigning one of two interventions at the church level, one "spiritual" and one "non-spiritual", then measuring change in knowledge between baseline and immediately after. ${ }^{25} \mathrm{PCa}$ knowledge increased $21 \%$ (from $71 \%$ to $92 \%$ ) in the spiritual arm, and increased $26 \%$ (from $72 \%$ to $98 \%$ ) in the non-spiritual arm. In the second RCT, "early" and "delayed" intervention groups received a culturally-tailored, multipart intervention including a videotape, printed pamphlet, and question answering session. ${ }^{31}$ The "early" intervention group attended an educational seminar at baseline, while the "delayed" intervention group attended the seminar at 3 months. The "early" intervention showed no difference from the "delayed" intervention on $\mathrm{PCa}$ knowledge at 3 months.

The remaining studies were pre-/post-assessments or cohort studies. Drake et al. designed an intervention based on the Ottawa Decision Support Framework and Health Belief Model. ${ }^{23}$ Knowledge increased on a $0-100$ scale by 25.7 points $(\mathrm{p}<0.001) .{ }^{23}$ Collins et al. found that, following a one-on-one counseling intervention, $\mathrm{PCa}$ knowledge increased by $41 \%$ from pre- to post-test, from 23 to $64 \%$ correct. Boehm et al. assessed the impact of an education session with AA speakers, one of whom was a physician and one a PCa survivor on PCa knowledge. ${ }^{20}$ Knowledge increased by $13 \%$, from 8.7 to 10.1 ( $\mathrm{p}<$ 0.001 ). Wilkinson et al. assessed a one-hour seminar using a 12-item questionnaire assessing knowledge of $\mathrm{PCa}$, and found that knowledge increased by $47 \%$, $(26 \%$ to $73 \%, \mathrm{p}<0.0001) .{ }^{30}$ Powell et al. utilized an educational program with an outreach coordinator; an 11-item knowledge inventory score increased from 8.7 to 10.1 (p $<0.0005)$, a $13 \%$ increase. $^{32}$ Carter et al assessed a 13 module intervention taught by trained men to AA men; knowledge was statistically-significantly higher in 13 of 17 areas, but percentage increases are not calculable. ${ }^{21}$

Printed Materials/Booklets $(\boldsymbol{n}=2)$. Taylor et al. conducted a 3-arm RCT comparing 1) a booklet intervention, 2) a videotape intervention, and 3) control. $^{28}$ The primary outcome was PCa knowledge differences, measured using an 11-item scale developed from the NCI Cancer Facts Sheet on $\mathrm{PCa}$ testing. $\mathrm{PCa}$ knowledge scores in the booklet arm increased from 7.2 to $9.2(\mathrm{p}<0.0001)$, an increase of $18 \%$ in the proportion correct (from $66 \%$ to $84 \%$ ). Similarly, in the videotape arm, knowledge scores increased relative to the control arm from 7.2 to $8.5(\mathrm{p}<0.0001)$, an $11 \%$ improvement in the proportion correct (from $66 \%$ to $77 \%$ ). Stephens et al. used an RCT to assess a printed decision aid for PCa screening. ${ }^{27}$ On a 24-item PCa knowledge scale, scores for AA increased from 17.5 to $20.6(\mathrm{p}<0.001)$, an increase of $13 \%$ in the proportion correct (from $73 \%$ to $86 \%$ ), with unchanged scores in the control arm (17.0 pre-test vs. 17.5 post-test; $p$-value "not significant"). ${ }^{27}$

Telephone/Videotape/DVD Interventions $(n=1)$. Barber et al. reported results from their existing PCa Early Detection Program surveying knowledge and attitudes about PCa before and after the program was implemented. ${ }^{19}$ Knowledge scores for the group increased from 4.6 to $5.8, \mathrm{p}<0.01$ ), a $16 \%$ increase in the proportion correct (from $66 \%$ to $83 \%$ ).

Web-based Interventions $(\boldsymbol{n}=2)$. Weston et al. evaluated a computer-assisted intervention by measuring pre-/post-test knowledge using an 18-item questionnaire from the National Cancer Institute (2002). ${ }^{29}$ PCa knowledge scores increased from 11.2 to 14.8 ( $\mathrm{p}<0.001)$, a $20 \%$ increase in the proportion correct (from $62 \%$ to $82 \%$ ). Ellison et al. conducted an RCT of a web-based decision aid and measured knowledge with a validated 12-item PCa knowledge test. ${ }^{24} \mathrm{PCa}$ knowledge scores were 6.78 for the usual care group and 7.67 for the decision aid group, a difference of $7.4 \%(p=0.0017)$.

Self-efficacy for Decision Making ( $\boldsymbol{n}=3$ ). Of the 14 studies of PCa screening, there were 2 papers assessing changes in 
self-efficacy for decision making in addition to knowledge changes, ${ }^{23,25}$ all using educational interventions. There was an additional study that evaluated self-efficacy for decision making alone. ${ }^{18}$

Educational Programs $(\boldsymbol{n}=2)$. Drake et al. created a theorydriven "road map" educational intervention for improving comfort, control and knowledge for PCa screening, and assessed outcomes immediately after. ${ }^{23}$ It was assessed with an 11-item decision self-efficacy scale, with scores ranging from 0 to 100 . The self-efficacy score increased from 84 to $93(\mathrm{p}=0.025)$, a $9 \%$ overall increase. Holt investigated a spirituality-based intervention (as described above) and found that self-efficacy for IDM significantly increased (mean $=0.55$ in the intervention arm versus mean $=0.44$ in the control arm; $\mathrm{p}<0.05){ }^{25}$

Printed Materials/Booklets $(\boldsymbol{n}=\mathbf{1})$. Kripalani et al. investigated the likelihood of a discussion about $\mathrm{PCa}$ in screening-eligible men, $90 \%$ of whom were AA. ${ }^{18}$ Patients were randomized to receive a patient education handout, a "cue" handout to ask about PCa, or a generic handout (control), and the likelihood of a discussion with the physician was recorded. Compared with the control group's likelihood of a conversation (37.3\%), the "cue" handout increased the likelihood the most (58.0 \%; OR: 2.39[1.26-4.52]), but the education handout also significantly increased the likelihood (50.0 \%; OR: 1.92[1.01-3.65]).

\section{Interventions to Improve Outcomes for Prostate Cancer Survivors}

Quality of Life ( $Q O L)(n=3)$. The interventions designed to improve QOL in minority cancer survivors all showed statistically significant improvements. ${ }^{33-35}$

Educational Programs $(\boldsymbol{n}=2)$. Two studies, both by Penedo et al., focused on Hispanic men following treatment for localized $\mathrm{PCa}^{21,22}$ One study captured a racially/ethnically diverse group (34\% Hispanic, $22 \% \mathrm{AA}$, $35 \%$ white), ${ }^{33}$ and the other was conducted in Hispanic men only. ${ }^{34}$ In both, a 10-week Cognitive-Behavioral Stress Management course ${ }^{36}$ was compared to a 1 day seminar with a control condition. QOL was measured using the 27item Functional Assessment of Cancer Therapy-General (FACT-G) Scale, assessing QOL across 4 domains (physical, social/family, emotional and functional). In the mixed ethnicity study, the intervention significantly improved mean QOL in the intervention group (87.31 12.41 vs. $90.48 \pm 11.27 ; \mathrm{p}<0.01)$, but not in the control group $(89.37 \pm 14.30$ vs. $88.65 \pm 13.19 ; \mathrm{p}>0.10)$ In the Hispanic-only study, several domains of QOL improved including: total QOL $(82.35 \pm 14.02$ vs.87.22 $\pm 13.21 ; \mathrm{p}=$
$0.05)$; physical well-being ( $23.98 \pm 4.51$ vs. $25.51 \pm 3.80$; $\mathrm{p}=$ $0.002)$; emotional well-being (19.24 \pm 4.04 vs. $20.73 \pm 3.31$; $\mathrm{p}=0.04)$; and sexual functioning $(5.61 \pm 6.35$ vs. $7.78 \pm 6.79$; $\mathrm{p}=0.002){ }^{33}$

Telephone/Videotape/DVD Interventions $(n=1)$. In a third QOL study, Campbell et al. conducted an RCT of a telephonebased coping skills intervention and evaluated QOL in AA men and their partners. ${ }^{35}$ QOL was measured using the SF-36 for general QOL and a disease-specific symptom assessment, the Expanded Prostate Cancer Index Composite (EPIC). The intervention group scored higher than the control group on the SF-36 on the Mental Health sub-scale ( 24.8 vs. $25.2 ; \mathrm{p}<0.08)$ but not on the Physical Health sub-scale (27.5 vs. 26.1; $\mathrm{p}=$ $0.34)$. At 6 weeks, patients reported improved QOL related to bowel symptoms $(p<0.05)$ and hormonal symptoms $(p<0.05)$ on the EPIC. However, the overall SF-36 scale showed no significant change.

Symptom Management Self-efficacy $(n=2)$. Mishel et al. conducted two related RCTs assessing symptom management in both AA and white men, focused on improving selfefficacy for symptom management in men receiving treatment for localized $\mathrm{PCa}$ using a DVD/booklet/telephone intervention. ${ }^{37,38}$

Telephone/Videotape/DVD Interventions $(\boldsymbol{n}=2)$. The effect of an "uncertainty management" invention on symptom control, using weekly telephone conversations with nurses for 8 weeks, was assessed at baseline, 3 months, and 7 months. ${ }^{37}$ A 26-item, "Uncertainty in Illness" scale measured emotional distress in AA men with localized $\mathrm{PCa}$. In the intervention arm, they found improvement for managing uncertainty using problem solving $(8.02 \pm 1.28$ vs. $8.32 \pm 1.28 ; \mathrm{p}<0.05)$ and cognitive reframing $(7.70 \pm 1.37$ vs. $8.20 \pm 1.37 ; \mathrm{p}<0.005)$ at 4 months, but no additional improvement at 7 months. In a second study also led by Michel, the authors used a similar uncertainty management intervention to assess self-efficacy for $\mathrm{PCa}$ and treatmentrelated symptoms. ${ }^{38}$ The intervention improved problemsolving ability $(\mathrm{p}=0.05)$. This effect persisted at 1 month, but disappeared at 3 months. No other differences were found.

\section{DISCUSSION}

The majority of interventions for ethnic/racial minority men, including both screening-eligible and treatment populations, improved some aspect of IDM or QOL for PCa. We draw the following conclusions from the studies: First, all but one relevant intervention for screeningeligible ethnic minority men increased $\mathrm{PCa}$ knowledge. Second, all relevant interventions improved self-efficacy for decision making about screening or symptom manage- 
ment. Third, educational programs showed the greatest percentage increase in knowledge across studies. Fourth, all culturally-sensitive psychosocial interventions in ethnic minorities successfully improved post-PCa treatment QOL and symptom management, at least in the short term. However, the overall magnitude of effect of one intervention compared with another was difficult to ascertain because of differences in interventions, differences in outcome measures, variations in patient populations and variations in study design, study duration, and analytical methods.

As hypothesized, shared decision making programs in ethnic minorities significantly increased PCa knowledge, at least in the short term. Interventions to improve knowledge about $\mathrm{PCa}$ in predominantly white, low-risk populations decrease PSA testing. ${ }^{39-42}$ It is unclear what effect knowledge interventions have on rates of PSA testing in minority populations. Educational materials in multiple formats-including educational programs, printed materials, telephone, DVD and web-based presentations-all facilitate IDM. Educational programs had the largest increases in PCa knowledge scores compared with printed materials/booklets, telephone/videotape/ DVD, and web-based interventions. Only one study reported the effect of specific, competing interventions on PCa knowledge; printed materials were shown to be slightly more effective than videotapes for improving and retaining PCa knowledge at 1 month. ${ }^{28}$ Studies generally assessed changes in $\mathrm{PCa}$ knowledge on the same day the intervention ended. Long term effects of interventions in screening-eligible populations are unknown.

Only 5 studies tested interventions to improve $\mathrm{PCa}$ treatments to reduce disparities. This lack of research is a major concern, particularly with a likely future decrease in PCa screening rates and a renewed emphasis on treatment only for symptomatic PCa. In the studies we identified, all culturally-sensitive cognitive behavioral interventions targeting treatment-related complications in ethnic minorities improved QOL. Most men treated for PCa survive many years and suffer from treatment-related complications. ${ }^{43}$ Managing the disease and its treatments long-term is even more crucial for ethnic minorities as they are less likely to discuss treatment-related complications with their physicians,${ }^{44}$ potentially leading to poorer QOL outcomes following treatment for $\mathrm{PCa}^{33-35}$ In addition, ethnic minority men, who are more likely to be in lower socioeconomic categories, typically have worse QOL outcomes and longer recovery periods from $\mathrm{PCa}$ treatments. For instance, Litwin et al. found that compared with whites, non-whites were much less likely to return to pretreatment level of physical (55\% vs. $90 \%$ ) and social (72\% vs. $91 \%$ ) functioning 1 year post-prostatectomy. ${ }^{45}$ In advanced $\mathrm{PCa}$, men in underserved populations had faster QOL declines on SF-36 scores. ${ }^{46}$ Given the high prevalence of
$\mathrm{PCa}$ in these men, it is critical to develop interventions that improve their QOL related to treatment. ${ }^{47}$

\section{LIMITATIONS}

Our review has several limitations. For historical reasons, most interventions were targeted to increasing PSA screening rates, which was an accepted goal during the time of most of these studies. However, we focused on the impact on IDM, which remains the central tenant of $\mathrm{PCa}$ management. Further, we did not assess for a risk of publication bias. A meta-analysis of the studies was not feasible given the lack of consistency in interventions, variability in follow-up intervals and outcomes across studies. Analysis of which intervention yielded the most improvement in PCa knowledge score is hampered by use of different knowledge scales across studies, lack of validity of several $\mathrm{PCa}$ knowledge scales, variability in study quality as assessed by Cochrane-modified DB scores, variation in the type of $\mathrm{PCa}$ knowledge provided within specific interventions, and lack of information on the effect of individual components of multi-faceted interventions on a specific outcome. Also, almost all of the studies assessed these outcomes on the same day as the interventions, and it is not clear that such knowledge would be retained after longer periods of time.

\section{CONCLUSIONS}

Overall, despite the increased risk of high-risk $\mathrm{PCa}$ in minority men, we found disappointingly few articles in the last 25 years on interventions to reduce those disparities. While screening for PCa has fallen out of favor, the core management strategy for $\mathrm{PCa}$ remains IDM. Appropriate interventions have consistently improved knowledge, increased self-efficacy, and raised QOL-all core components of IDM. ${ }^{48}$ Educational programs were the most effective intervention for improving knowledge among screening-eligible minority men. Cognitive behavioral strategies improved QOL for minority men treated for localized PCa. Going forward, it is essential that we create a much stronger evidence base for the use of such interventions to improve IDM across the clinical spectrum for $\mathrm{PCa}^{49}$

Funding Source: Support for this publication was provided by a grant from the Robert Wood Johnson Foundation's Finding Answers: Disparities Research for Change program.

Prior Presentation: Poster presented at Presidential Poster Session, American Geriatrics Society, Annual Meeting, 2011. 
Conflict of Interest: The authors declare that they do not have a conflict of interest.

Corresponding Author: William Dale, MD, PhD; Department of Medicine, Section of Geriatrics, Palliative Medicine, University of Chicago Medical Center, 5841 S. Maryland Avenue, MC6098, Chicago, IL 60637, USA (e-mail: wdale@medicine.bsd.uchicago.edu).

\section{REFERENCES}

1. Jemal A, Siegel R, Xu J, Ward E. Cancer statistics, 2010. CA Cancer J Clin. 2010;60(5):277-300.

2. Williams H, Powell IJ. Epidemiology, pathology, and genetics of prostate cancer among African Americans compared with other ethnicities. Methods Mol Biol. 2009;472:439-53.

3. Gwede CK, McDermott RJ. Prostate cancer screening decision making under controversy: implications for health promotion practice. Health Promot Pract. 2006;7(1):134-46.

4. Minardi D, Galosi AB, Recchioni A, Giammarco L, Polito M, Muzzonigro G. Diagnostic accuracy of percent free prostate-specific antigen in prostatic pathology and its usefulness in monitoring prostatic cancer patients. Urol Int. 2001;67(4):272-82.

5. Eschenbach A, Ho R, Murphy G, Cunningham M, Lins N. American Cancer Society Guideline for the Early Detection of Prostate Cancer: Update 1997. CA Cancer J Clin. 1997;47:261-4.

6. U.S. Preventive Services Task Force. Screening for Prostate Cancer: US Preventitive Services Task Force Recommendation Statement (DRAFT). 2011. http://www.uspreventiveservicestaskforce.org/uspstf12/prostate/ draftrecprostate.htm. Accessed on April 02, 2012.

7. Guide to Clinical Preventive Services. United States Preventive Services Task Force. Alexandria, VA: International Medical Publishing; 2nd ed. 1996:119-34.

8. Klabunde CN, Potosky AL, Harlan LC, Kramer BS. Trends and black/ white differences in treatment for nonmetastatic prostate cancer. Med Care. 1998;36(9): 1337-48.

9. Chan E, McFall S, Byrd T, et al. A community-based intervention to promote informed decision making for prostate cancer screening among Hispanic American men changed knowledge and role preferences: a cluster RCT. Patient Educ Couns. 2011;84(2):44-51.

10. Barry MJ. Screening for prostate cancer-the controversy that refuses to die. N Engl J Med. 26 2009;360(13):1351-4.

11. Chu K, Tarone R, Freeman $\mathbf{H}$. Trends in prostate cancer mortality among black men and white men in the United States. Cancer. 2003;97(6).

12. Demark-Wahnefried W, Schildkraut JM, Iselin CE, et al. Treatment options, selection, and satisfaction among African American and white men with prostate carcinoma in North Carolina. Cancer. 1998;83 (2):320-30.

13. Dimah K. Prostate cancer among African American men: a review of empirical literature. J Afr Am Stud. 2003;7(1):28-46.

14. Tillman LC. Culturally sensitive research approaches: an AfricanAmerican perspective. Educ Res. 2002;31(9):3-12.

15. Andersen BL. Behavioral outcomes following psychological interventions for cancer patients.J Consult Clin Psychol. 2002;70(3):590610

16. Moher D, Liberati A, Tetzlaff J, Altman DG. Preferred reporting items for systematic reviews and meta-analyses: the PRISMA statement. PLoS med. 2009;6(7):e1000097.

17. Downs SH, Black N. The feasibility of creating a checklist for the assessment of the methodological quality both of randomised and nonrandomised studies of health care interventions. J Epidemiol Community Health. 1998;52(6):377.

18. Kripalani S, Sharma J, Justice E, et al. Low-literacy interventions to promote discussion of prostate Cancer: a randomized controlled trial. Am J Prev Med. 2007;33(2):83-90.

19. Barber K, Shaw R, Folts M, et al. Differences between African American and Caucasian men participating in a community-based prostate cancer screening program. J Community Health. 1998;23(6):441-51.

20. Boehm S, Coleman-Burns P, Schlenk E, Funnell M, Parzuchowski J, Powell I. Prostate cancer in African American men: increasing knowledge and self-efficacy. J Community Health Nurs. 1995;12 (3): 161-9.
21. Carter VL, Tippett F, Anderson DL, Tameru B. Increasing prostate cancer screening among African American men. J Health Care for Poor Underserved. 2010;21(3A):91-106.

22. Collins M. Increasing prostate cancer awareness in African American men. Oncol Nurs Forum. 1997;24(1):91.

23. Drake B, Shelton R, Gilligan T, Allen J. A church-based intervention to promote informed decision making for prostate cancer screening among African American men. J Natl Med Assoc. 2010;102(3):164-71.

24. Ellison G, Weinrich S, Lou M, Hongyan X, Powell I, Baquet C. A randomized trial comparing web-based decision aids on prostate cancer knowledge for African-American men. J Natl Med Assoc. 2008;100 (10): 1139-45.

25. Holt C, Wynn T, Litaker M, Southward P, Jeames S, Schulz E. A comparison of a spiritually based and non-spiritually based educational intervention for informed decision making for prostate cancer screening among church-attending African-American men. Urol Nurs. 2009;29 (4):249.

26. Powell I, Heilbrun L, Littrup P, et al. Outcome of African American men screened for prostate cancer: The Detroit Education and Early Detection Study. J Urol. 1997;158(1):146-9.

27. Stephens RL, Xu Y, Volk RJ, et al. Influence of a patient decision aid on decisional conflict related to PSA testing: A structural equation model. Health Psychol. 2008;27(6):711-21.

28. Taylor K, Davis J, Turner R, et al. Educating African American men about the prostate cancer screening dilemma: a randomized intervention. Cancer Epidemiol Biomarkers Prev. 2006;15(11):2179.

29. Weston R, Weston P, Futterman R, et al. Effectiveness of a modified computer assisted instructional tool in the dissemination of prostate cancer information to men of African descent through black churches. J Afr Am Stud. 2007;11(2): 140-56.

30. Wilkinson S, List M, Sinner M, Dai L, Chodak G. Educating AfricanAmerican men about prostate cancer: impact on awareness and knowledge. Urology. 2003;61(2):308-13.

31. Husaini B, Reece M, Emerson J, Scales S, Hull P, Levine R. A churchbased program on prostate cancer screening for African American men: reducing health disparities. Ethn Dis. 2008;18:179-84

32. Powell I, Gelfand D, Parzuchowski J, Heilbrun L, Franklin A. A successful recruitment process of African American men for early detection of prostate cancer. Cancer. 1995;75(S7):1880-4.

33. Penedo F, Dahn J, Molton I, et al. Cognitive behavioral stress management improves stress management skills and quality of life in men recovering from treatment of prostate carcinoma. Cancer. 2004;100 (1): 192-200.

34. Penedo F, Traeger L, Dahn J, et al. Cognitive behavioral stress management intervention improves quality of life in Spanish monolingual Hispanic men treated for localized prostate cancer: results of a randomized controlled trial. Int J Behav Med. 2007;14(3):164-72.

35. Campbell L, Keefe F, Scipio C, et al. Facilitating research participation and improving quality of life for African American prostate cancer survivors and their intimate partners. Cancer. 2007;109(S2):414-24.

36. Antoni MH. Stress management effects on psychological, endocrinological, and immune functioning in men with HIV infection: empirical support for a psychoneuroimmunological model. Stress: Int $\mathrm{J}$ Biol Stress. 2003;6(3): 173-88

37. Mishel M, Belyea M, Germino B, et al. Helping patients with localized prostate carcinoma manage uncertainty and treatment side effects. Cancer. 2002;94(6):1854-66.

38. Mishel M, Germino B, Lin L, et al. Managing uncertainty about treatment decision making in early stage prostate cancer: a randomized clinical trial. Patient Educ Couns. 2009;77(3):349-59.

39. Partin MR, Nelson D, Radosevich D, et al. Randomized trial examining the effect of two prostate cancer screening educational interventions on patient knowledge, preferences, and behaviors. J Gen Intern Med. 2004;19(8):835-42.

40. Volk RJ, Hawley ST, Kneuper S, et al. Trials of decision aids for prostate cancer screening: a systematic review. Am j prev med. 2007;33 (5):428-34. e411

41. Evans R, Joseph-Williams N, Edwards A, et al. Supporting informed decision making for prostate specific antigen (PSA) testing on the web: an online randomized controlled trial. J Med Internet Res. 2010;12(3).

42. Handley MR, Stuart ME. The use of prostate specific antigen for prostate cancer screening: a managed care perspective. J Urol. 1994;152(5 Pt 2): 1689.

43. Eton DT, Lepore SJ. Prostate cancer and health-related quality of life: a review of the literature. Psycho-Oncol. 2002;11(4):307-26. 
44. Penedo FJ, Dahn JR, Shen BJ, Schneiderman N, Antoni MH. Ethnicity and determinants of quality of life after prostate cancer treatment. Urology. 2006;67(5):1022-7.

45. Litwin MS, Flanders SC, Pasta DJ, Stoddard ML, Lubeck DP, Henning JM. Sexual function and bother after radical prostatectomy or radiation for prostate cancer: multivariate quality-of-life analysis from CAPSURE. Urology. 1999;54(3):503-8.

46. Melmed GY, Kwan L, Reid K, Litwin MS. Quality of life at the end of life: trends in patients with metastatic prostate cancer. Urology. 2002;59 (1):103-9.
47. Lepore SJ, Helgeson VS, Eton DT, Schulz R. Improving quality of life in men with prostate cancer: a randomized controlled trial of group education interventions. Health Psychol. 2003;22 (5): 443 .

48. Kon AA. The shared decision-making continuum. JAMA: J Am Med Assoc. 2010;304(8):903-4.

49. Sajid S, Mohile SG, Szmulewitz R, Posadas E, Dale W. Individualized decision-making for older men with prostate cancer: balancing cancer control with treatment consequences across the clinical spectrum. Semin Oncol. 2011;38(2):309-25. 